\title{
Droit et violence. Freud et Benjamin ${ }^{1}$
}

\begin{abstract}
Alain Vanier
$\mathrm{Au}$ couple droit (Recht) et puissance (Macht) avancé par Einstein, Freud propose de substituer le couple droit (Recht) et violence (Gewalt), et entend montrer comment ces deux termes, qui semblent aujourd'hui s'opposer, ont en fait une origine commune, " l'un s'est développé à partir de l'autre ». Le terme Gewalt en allemand signifie violence, force, mais aussi autorité, pouvoir et veut dire aussi bien pouvoir judiciaire (richterliche Gewalt) que puissance paternelle (ou parentale) (elterliche Gewalt). Il peut désigner un attribut des institutions sociales, ainsi geistliche Gewalt, le pouvoir spirituel de l'Église, et Staatgewalt, le pouvoir de l'État.

«Initialement, dans une petite horde humaine, c'est la force musculaire la plus grande qui décidait à qui quelque chose devait appartenir ou de qui la volonté devait être exécutée », écrit Freud. Ainsi, à l'origine, la loi s'impose par la force, la force du corps. Cette force est mue par une volonté qui s'applique à l'autre concernant un objet ou à l'autre comme objet. Le pouvoir comme technique est développement et déplacement de la puissance corporelle. Dans un temps ultérieur seulement, la paix sociale peut s'organiser par « le surmontement de la violence par transfert de la puissance à une unité plus grande ». Cette unité est celle de la communauté en tant que communauté d'intérêt qui

Alain Vanier, psychanalyste, Professeur de Universités, directeur du Centre de Recherches Psychanalyse et Médecine (CRPM) de l'Université Paris Diderot - Paris 7

${ }^{1}$ Ce texte a fait l'objet d'une première parution dans Le Bloc-notes de la psychanalyse, $\mathrm{n}^{\circ} 18$, « La guerre, la pulsion de mort », 2003
\end{abstract}


s'instaure dans un groupe humain. Dans ce mouvement, le groupe dominant substitue, à un moment donné, l'esclavage à la mise à mort. Le corps de l'autre peut servir les intérêts économiques de celui qui impose sa loi. Ce faisant, celui-ci, le vainqueur, « abandonne une part de sa propre sécurité » car le vaincu, vivant, constitue une menace. La stabilité légale et la tranquillité qu'elle produit n'est obtenue que par la substitution du rapport entre vainqueur et vaincu par ceux de maître et d'esclave. "Le droit de la communauté devient alors l'expression des rapports de puissance inégaux en son sein, les lois seront faites par et pour les dominants. " La paix contient donc la guerre comme son fondement et n'est plus que l'expression d'une violence collective qu'imposent les vainqueurs aux vaincus, l'expression d'une violence en puissance. Sur cette base, se crée une communauté organisée fondamentalement par la contrainte, et dont la cohésion est assurée par des phénomènes d'identification, perçus comme des sentiments. Cette violence qui a dépossédé le vaincu et qui assure la base de la tranquillité sociale, oblige le vainqueur, pour sa sécurité, à remettre en circuit un peu de ce qu'il a gagné pour maintenir en vie le corps de l'esclave, son capital, afin que celui-ci puisse accroître encore la quantité des biens que possède le vainqueur. Sous un certain angle, cette paix sociale est une guerre qui répète inlassablement la dépossession originelle par la violence dont le vaincu fut l'objet. En effet, celui-ci ne cesse pour partie d'être dépossédé de ce qui lui revient et de ce qu'il produit. Aliénation du travail et plusvalue, selon Marx, que Lacan propose de lire comme plus-de-jouir. Cette jouissance originelle, cette violence, circule de façon canalisée et régulée dans le lien social, c'est la fonction du droit. Chacun peut alors avoir l'espoir qu'il récupère ou qu'il va récupérer un peu de ce dont il est spolié.

Dans ces quelques pages, Freud édifie un mythe qui est en quelque sorte le prolongement de Totem et tabou. La lecture de l'histoire qu'il met en jeu est un nouage et une dialectique entre deux pôles en tension. La tendance à l'union (Einigung) se manifeste déjà dans le meurtre, puis dans le repas totémique des frères après la mort de l'Urvater, du père de la horde. Cette tendance à constituer des unités de plus en plus grandes, ou à unifier des éléments multiples en les agrégeant, que Freud nomme Éros, entre en conflit avec un pôle de destructivité qui s'y oppose. Mais l'une des deux motions ne l'emporte jamais totalement sur l'autre. La violence est originaire et l'union en triomphe : « La violence est brisée par 
l'union. » Mais cette violence fonde la puissance de posséder ce que l'autre possède, voire lui-même et sa force de travail ; la violence est origine du droit. L'union ne fait pas disparaître la violence, mais la déplace, en effet, à ce moment, « ce n'est plus la violence d'un individu qui s'impose, mais celle de la communauté »); la violence garantit le droit.

Cette stabilité est toujours menacée, car la paix est ainsi continuation d'une violence qui s'exprime autrement, et les lois faites par et pour les dominants « concéderont peu de droit aux soumis ». D'autre part, dans ce jeu constant entre Éros et pulsion de mort, un certain paradoxe apparaît. Il faut « concéder que la guerre ne serait pas un moyen inapproprié pour instaurer la paix « éternelle » tant désirée, car elle est en mesure de créer ces grandes unités à l'intérieur desquelles un pouvoir central fort rend de nouvelles guerres impossibles ». Mais à cette perspective, s'oppose quelque chose comme un principe de limitation, d'entropie. L'unité absolue n'est pas réalisable. En effet, plus l'agrégation des unités sera grande, plus elle sera en même temps fragile par manque de cohésion des parties unies par la violence. Ce que la violence fonde, comme tendance à constituer des unités de plus en plus grandes, a pour Freud une limite, certainement inspirée par l'exemple de l'empire des Habsbourg, limite qui, pour lui, est la fragilité de l'union des parties à mesure que le nombre des parties est plus grand. Ce modèle s'applique aussi à la situation présente, à l'heure de cet échange de lettres avec Einstein, la Société des Nations pouvant être conçue comme ce pouvoir central régulateur et protecteur de la paix, à la condition qu'il ait la puissance requise. Il faut, pour maintenir la cohésion communautaire, deux facteurs : un système d'identifications entre les membres et la contrainte de la violence. Avec quelques décennies de recul, les enjeux contemporains, par exemple ce qu'on appelle globalisation ou mondialisation et les effets de ségrégation qu'elle entraîne, n'obligent-ils pas à reconsidérer la question d'une autre manière?

« Le droit est la puissance d'une communauté », le droit est encore et toujours de la violence. Et Freud de conclure : « C'est une erreur de calcul de ne pas considérer que ce qui est droit était à l'origine violence brute et qu'aujourd'hui encore il ne peut se passer du soutien de la violence. » Freud refuse d'identifier la violence, aussi bien que le mal, à la pulsion de mort, le bien et la paix à la pulsion de vie, Éros. Leur nouage est fondamental, voire inextricable, puisque la pulsion d'amour, par exemple, 
a besoin aussi de la pulsion d'emprise. Le dégagement tardif de ces deux pulsions dans l'histoire de la psychanalyse vient de la difficulté qu'il y a à les isoler. Mais peut-être aussi de la nécessité d'un moment dans l'histoire qui permette de les penser, d'une spécificité actuelle qui, au-delà ou à partir du mythe proposé par Freud, en présente plus lisiblement l'occurrence.

La violence brute originelle et la violence aujourd'hui nécessaire sont-elles de même nature ? Le droit, une fois fondé, se pose la question de la légitimité de la violence, c'est-à-dire du rapport non plus entre la violence et le droit, mais entre la violence et la justice. Ainsi, la violence, relevée par Freud, du soumis à l'égard du dominant (Herrschenden) estelle légitime au regard, par exemple, de la violence qui serait justifiée par une volonté de conquête ou d'asservissement à l'égard d'un groupe voisin?

Walter Benjamin tente de distinguer plusieurs statuts de la violence (Gewalt), et les distinctions qu'il introduit peuvent nous être utiles. Il rappelle d'abord l'opposition entre droit naturel et droit positif, la distinction classique entre fins et moyens, etc. Mais il s'intéresse, dans cette perspective, à la question de la violence, en particulier à la distinction entre une violence légitime et une violence qui ne l'est pas. Cette distinction est néanmoins problématique. Il évoque le statut de la grève. La grève est-elle une violence puisqu'elle consiste en une suspension de l'activité ? À l'extrême, la grève générale, dans une perspective révolutionnaire, peut-elle être considérée comme une non-violence? Comment penser sa légitimité ? Sans développer plus avant les commentaires fort riches de Walter Benjamin qui nous entraîneraient sur d'autres chemins, arrêtons-nous sur cette distinction des violences, d'une part, la violence qui fonde le droit, et, d'autre part, celle qui conserve le droit. Ces deux violences, souligne Benjamin, se retrouvent dans une institution de l'État moderne : la police. C'est bien là ce qui nous intéresse : l'articulation contemporaine entre droit et violence. Foucault aussi remarquait que la police était une institution récente. La police moderne, pour lui, témoigne d'un changement historique des rapports entre pouvoir et individus. "La société et les hommes en tant qu'êtres sociaux, individus forts de tous leurs rapports sociaux : tel est désormais le véritable objet de la police. » Pour Foucault, cette émergence de la police coïncide avec le moment où « le gouvernement se met à s'occuper 
des individus, en fonction de leur statut juridique, certes, mais aussi en tant qu'hommes, êtres vivants qui travaillent et commercent ». Mais aussi, l'émergence d'un nouveau statut du vivant fondé sur la science. En effet, Foucault repérait cette inflexion dans l'apparition des premiers grands programmes d'hygiène des populations. Ainsi l'État veille sur les hommes en tant que population, "sa politique est, en conséquence, nécessairement une biopolitique ». Pour les auteurs des XVII ${ }^{\mathrm{e}}$ et $\mathrm{XVIII}^{\mathrm{e}}$ siècles, police ne signifie pas nécessairement une institution, mais plutôt une technique de gouvernement propre à l'État. La police comme institution de l'État moderne a déjà été relevée par Benjamin. Pour lui, le caractère de l'autorité de la police est ignoble. Son ignominie tient à l'absence de séparation entre deux violences, celle qui fonde le droit et celle qui conserve le droit. En effet, la police intervient dans des cas où la situation juridique n'est pas claire. Elle ne conserve alors pas simplement le droit en tant qu'il est écrit, elle est, dans son action même, fondatrice du droit. Il note d'ailleurs que l'esprit de police fait moins de ravages quand, dans la monarchie absolue, «elle représente la violence du souverain » en qui sont unis les pouvoirs législatif et exécutif. Cette union, au nom d'une instance transcendante au-delà du souverain, mais qui le fonde, est aussi confusion, recouvrement entre droit et action, et suture de toute béance le sens.

La latence de la violence permet l'institution juridique. Benjamin prend l'exemple des modes de liquidation des conflits sans violence. Il en existe, dit-il, non pas quand il y a des rapports d'homme à homme, mais quand ces rapports font l'objet d'une médiation. Le dialogue est pour lui une technique d'accord civil, le symbolique possède un versant pacifiant, pourrions-nous dire. Et pour lui, l'exclusion de toute violence dans la sphère privée peut se lire dans l'impunité du mensonge. Ce domaine est celui de «l'entente qui est celui du langage ». Et lorsque le droit légifère dans la sphère privée, il s'affaiblit. Ainsi, lorsqu'il interdit la tromperie, il limite l'emploi des moyens non violents. Ces dispositions juridiques produisent au contraire des effets violents, c'est-à-dire que, dans ce cas, le droit perd lui-même confiance dans sa propre violence. Ces remarques sont tout à fait d'actualité : en légiférant sur ce point, c'est une certaine dimension de la parole dans son rapport à la vérité qui est évacuée. Ce mensonge que Lacan désigne comme réellement symbolique, c'est-à-dire comme du symbolique inclus dans le réel, ce que l'on doit entendre sur le 
fond du proton pseudos hystérique relevé par Freud, de ce premier mensonge qui est refoulement.

La fondation du droit est fondation du pouvoir. Ainsi, Benjamin peut écrire : «Si la justice est le principe de toute finalité divine, le pouvoir est le principe de toute fondation mythique du droit.» Il introduit une nouvelle distinction et oppose alors violence mythique et violence divine. La violence divine est hors du droit, destructrice de droit, destructrice sans limites, elle lave de la faute, et n'est pas sanglante mais mortelle. Le sang, souligne Benjamin, est symbole de la « simple vie » et c'est pourquoi la violence mythique est une violence sanglante exercée en sa propre faveur contre la vie pure et simple, qui exige le sacrifice. À sa différence, la violence divine est violence pure, exercée en faveur du vivant contre toute vie, elle accepte le sacrifice. L'homme ne doit pas être confondu avec la simple vie qui est en lui. Benjamin pense que le dogme qui affirme le caractère sacré de la vie est récent. Il s'agit pour lui de l'ultime « égarement de la tradition occidentale affaiblie qui cherche dans le cosmologiquement impénétrable le sacré qu'elle a perdu». En effet, «ce qui est ici qualifié de sacré est ce que l'ancienne pensée mythique désignait comme porteur de culpabilité : le simple fait de vivre ». Car en tout point Dieu s'oppose au mythe. Le mythe est une entrave et si son règne est battu en brèche, alors le nouveau n'est pas lointain. Il faut ainsi, dit Benjamin, rejeter toute violence mythique comme violence fondatrice du droit, qu'on peut appeler aussi violence discrétionnaire, mais aussi bien la violence conservatrice de droit, c'est-à-dire la violence administrée, qui est au service de la précédente. Benjamin termine sur cette formule : « La violence divine, qui est insigne et sceau, non point jamais moyen d'exécution sacrée, peut être appelée souveraine. » Dans le commentaire qu'il fait de ce texte, Derrida lie la violence mythique comme référée à la culture grecque et la violence divine au judaïsme. Derrida interprète cette opposition de Benjamin comme une opposition de l'histoire au mythe. «Pour schématiser, il y aurait deux violences, deux Gewalten concurrentes : d'un côté, la décision (juste, historique, politique, etc.), la justice au-delà du droit et de l'État, mais sans connaissance décidable; de l'autre, il y aurait connaissance décidable et certitude dans un domaine qui reste structurellement celui de l'indécidable, du droit mythique et de l'État. D'un côté, la décision sans certitude décidable, de l'autre, la certitude de l'indécidable mais sans décision. » 
Lacan distingue aussi les traditions grecques et juives, et, plus précisément le Dieu des philosophes et le Dieu du monothéisme. Le Dieu des philosophes est l'Autre de la structure originelle, celui qui est un. C'est le Dieu du mythe. Le Dieu du monothéisme n'est pas un, il ne dit pas qu'il est le seul Dieu. Les autres dieux ne sont pas niés, ils sont ailleurs que là où il est. Il est seulement le Dieu qui parle et qui dit : « Je suis ce que je suis. » Il introduit la dimension de la Révélation, celle de « de la parole comme porteuse de vérité » et la distinction, fondamentale, entre vérité et savoir, car «ailleurs la place de la vérité est remplie [...] à l'occasion par des mythes ». Cette énonciation fait trou, irrésorbable.

Cette violence divine, selon Benjamin, est insigne, sceau, marque, écriture, écriture du nom de ce dieu dont le nom s'écrit mais ne se prononce plus et ne peut donc pas se savoir. Elle est béance dans le savoir, elle figure la part hors droit, non reprise par le lien social, le discours, elle est ce reste originaire qui, aujourd'hui, peut se repérer et semble pouvoir faire retour comme instant, comme coupure absolue. Le texte de Benjamin sur la critique de la violence est de 1920, il trouve un écho dans des textes plus tardifs qui prolongent et remanient ces propositions.

Dans sa réponse à Einstein, Freud développe un certain nombre de remarques sur la pulsion de mort, en soulignant qu'elle ne saurait être assimilée ni au mal ni même à la guerre. Lacan soulignait que la guerre était une des formes du commerce interhumain et qu'elle était profondément enracinée dans la structure de l'échange dont elle est une des modalités. Le trajet même de la pulsion, tout comme le statut de l'objet dans l'échange, suppose, à son terme, une certaine volatilisation, destruction de cet objet, ne serait-ce que du fait de son inadéquation foncière, due à son caractère fondamentalement perdu. Trois modes de lecture ou trois projections de la pulsion de mort sont possibles selon les registres Imaginaire, Symbolique et Réel. Dans une première approche, Lacan met l'accent sur la tension ressentie par le sujet, par l'infans, face à son image dans le miroir. Face à cette image pour laquelle il est aimé, mais qui lui est en même temps étrangère et dans laquelle il s'aliène, le sujet se trouve dans une situation sans issue que Lacan référait à la situation duelle qui peut s'exprimer par le « ou bien... ou bien... » Le rapport strict à cette image, sans médiation, est mortel, ce que montre bien le mythe de Narcisse. C'est la parole qui, distinguant les positions 
respectives, sort le sujet de cette impasse. Ainsi l'agressivité, le rapport au semblable se déploie sur le fond de cette relation narcissique fondée sur l'exclusion. Si la dimension symbolique, avec la mise en jeu de la parole, sort le sujet de cette impasse, elle n'est pas exempte d'une dimension mortelle elle-même. Du point de vue du symbolique, si l'on reprend les moments du miroir, il y a un temps nécessaire où l'Autre est convié à fournir une médiation. Ce mouvement se figure par celui de l'enfant se tournant vers la mère qui le porte devant le miroir pour lire dans son regard un assentiment, un signe de reconnaissance. Quand il se retourne ensuite vers cette image, la sienne, qui était déjà là, cette médiation lui échappe, «il n'y subsiste que cet être dont l'avènement ne se saisit qu'à n'être plus », qui est disparition de l'être du fait de la nomination même. Ainsi cette image, le sujet ne l'assume, comme corps imaginaire, que parce que l'Autre, une parole le lui décerne. Ce qui lui permet de résoudre la tension du rapport duel à sa propre image. En même temps, une autre aliénation s'instaure, aliénation au signifiant, et le sujet disparaît sous la nomination qui vient de l'Autre. Le signifiant est porteur d'une dimension mortelle, celle-là même qui s'effectue aussi dans la symbolisation primordiale. Le mot est le meurtre de la chose, a pu reprendre Lacan, la dimension éternisante et mortelle du signifiant est en jeu et se trouve au principe de l'automatisme de répétition. Signifiant qui est aussi ce à quoi se réduit le sujet, un nom propre sur une pierre tombale. Ce qu'il en est de cette instance, nommée par Freud pulsion de mort, se loge donc à plusieurs enseignes. Elle est aussi dépendante, dans ses manifestations, des éléments historiques et des remaniements qui affectent la subjectivité. Ainsi, l'émergence de la science moderne n'est pas sans effet. Lacan a pu dire que la pulsion de mort s'était réfugiée dans la physique moderne et j'ai pu déjà rapprocher cette formule de celle de Winnicott qui pensait que l'adolescence, comme problème noué à la question de la violence, venait précisément de la guerre froide qui ne permettait plus de régler et d'occulter le problème de l'adolescence, comme il l'était autrefois par une guerre par génération où l'on envoyait les adolescents défendre la patrie. L'impossibilité des guerres du fait de la puissance de destruction donnée par les armes que fournit la science a en effet changé la donne. Dans cette perspective, Lacan a souligné ces " conséquences du remaniement des groupes sociaux par la science, et nommément de l'universalisation qu'elle y introduit ». Marchés communs 
auxquels Lacan fait allusion à l'époque, globalisation, mondialisation d'aujourd'hui, auront imparablement sur le plan réel un corrélat dans la montée des ségrégations. L'un quelconque du sujet moderne, de ce sujet sans qualités, sa similarité biologique, l'appareillage de son corps par les produits de la technique, de la prothèse à l'automobile, annulent, en dévoilant sa facticité, le vieil ordre soutenu par une figure divine dans lequel le sujet, certes infantilisé selon Freud, trouvait sa place et son unicité. Lacan soulignait que ce problème pouvait se lire en termes d'une problématique de la jouissance, jouissance qui ne pouvait être située que de l'Autre. Mais, ajoutait-il, «c'est en tant que nous en sommes séparés ». Or, notre mode de jouissance moderne ne se situe plus que du plus-dejouir, $a$, et non du grand Autre qui l'organisait et la cadrait. Ainsi, la ségrégation se fonde d'un échec de la séparation.

Dans sa lettre à Einstein, Freud souligne comment la cohésion d'un groupe - il prend l'exemple des bolcheviques - ne se fonde que sur l'exclusion d'un autre qui polarise toute la haine. Il en vient à proposer sa propre utopie, fille de celle des Lumières : une vie pulsionnelle soumise à la dictature de la raison. Mais il est sans illusions.

Freud s'arrête sur la question posée par Einstein : pourquoi nous indignons-nous contre la guerre ? "Pourquoi ne l'acceptons-nous pas comme telle autre des nombreuses et cruelles nécessités de la vie ? » À côté de plusieurs autres éléments de réponse, Freud souligne quelque chose qui lui paraît propre à l'époque moderne. " C'est aussi que, dans sa configuration présente, la guerre ne donne plus l'occasion de réaliser le vieil idéal héroïque, et qu'une guerre future, par suite du perfectionnement des moyens de destruction, signifierait l'extermination de l'un ou peutêtre des deux adversaires. »

Pour commenter cette remarque de Freud, un nouveau détour par Walter Benjamin me semble utile. Il ne s'agira pas ici de développer le lien particulier que tisse Benjamin entre la théologie et le marxisme. Simplement peut-être faut-il indiquer que son messianisme est un messianisme paradoxal puisqu'en même temps plusieurs éléments dans les textes de Benjamin montrent qu'il s'agit d'un messianisme lié à un Dieu absent, et que c'est dans la dimension historique que l'humanité peut être son propre messie. Mais l'histoire est toujours l'histoire des vainqueurs; elle annule l'histoire des vaincus et, de ce fait, «il n'est 
aucun document de culture qui ne soit aussi document de barbarie ». Comment Benjamin conçoit-il la modernité ? On peut partir de ses considérations sur l'effet de la technique moderne sur l'œuvre d'art. Il remarque le fait que les outils techniques modernes permettent sa reproductibilité. «Ce processus a valeur de symptôme ; sa signification dépasse le domaine de l'art. On pourrait dire, de façon générale, que la technique de reproduction détache l'objet reproduit du domaine de la tradition. » L'œuvre d'art moderne, parce que reproductible, perd son aura. Cette perte de l'aura est un appauvrissement quant au sens de l'œuvre d'art, une perte de fascination due à l'unicité. Cette perte de l'aura peut être lue de deux manières : comme symptôme de décadence, d'une part, et, dans ce cas, l'issue peut être de restaurer la tradition. Mais ce moment historique est aussi le lieu possible d'une rédemption. En effet, ces moments d'inflexion de l'histoire sont des moments où se marque un lien particulier au temps. Ainsi Benjamin relève que, lors de la révolution de Juillet, les insurgés tirèrent sur les horloges de Paris. Il y a une dimension du temps actuel, de l'à présent, du maintenant (Jetztzeit), un moment où peut se récapituler toute l'histoire de l'humanité, et en particulier l'histoire, non advenue, refoulée ou forclose - ce qu'il conviendrait de préciser - des vaincus. Ces moments, rares, dans l'histoire sont ceux d'une possible rédemption. Le monde moderne en a fini, selon Benjamin, avec ses dieux et ses incarnations, ce qui le met dans une situation désespérée. Il est en attente de libération (Erlösung), ce qui devient possible pour lui car ce temps de rupture est aussi un temps d'arrêt. L'aura est à la fois un faux-semblant, un enchantement, et la marque du lien de l'œuvre d'art avec sa dimension cultuelle. Le religieux apparaît pour ce qu'il est : un pouvoir qui aliène. Mais le déclin de l'aura est aussi une dénonciation de "l'ensemble de l'histoire de l'humanité comme une seule et même tentative utopique visant à renouer avec l'origine. En finir avec l'aura, c'est donc affirmer l'arrêt, la suspension de toute illusion, en particulier celle de l'origine. »

Après avoir schématiquement situé la perspective, c'est le texte de Benjamin sur la théorie du fascisme allemand que je souhaiterais pouvoir mettre en regard de l'approche de Freud de la guerre moderne. La position de Benjamin sur la technique dans ce texte peut retenir notre attention. Loin de la condamner, il interroge sa place. "Toute guerre à venir sera aussi une révolte de la technique contre la condition servile dans laquelle 
elle est tenue. \ Dans ce texte, il renvoie dos à dos aussi bien les mystiques de la guerre que les pacifistes. Il s'agit du commentaire d'un livre d'Ernst Jünger, intitulé Krieg und Krieger (Guerre et guerriers), qu'il classe dans la catégorie de ces mystiques de la guerre. Peut-être avons-nous trop tendance aujourd'hui, en mettant, à juste titre sans doute, l'accent sur la catastrophe sans nom que fut la Seconde Guerre mondiale, à méconnaître la fonction de première dans la série que fut la Première Guerre mondiale. Ce livre de Jünger est une déclaration nostalgique qui en appelle au renouement avec le statut ancien des guerres avant la dernière, et, en particulier, avec la valeur de l'héroïsme. Il défend une idéologie de la guerre totalement périmée au regard de ce qui vient de se passer. « Jamais ils ne s'avisent que la bataille de matériel dans laquelle certains d'entre eux voient la plus haute révélation de l'existence, disqualifient les pauvres emblèmes de l'héroïsme », formule qui trouve un écho dans les propos de Freud. On a souvent fait de ces textes de Benjamin, celui-là entre autres, une anticipation où pouvait se lire le pressentiment de ce qui allait arriver : son insistance, ici, sur l'utilisation du gaz dans les combats de tranchée. La guerre a changé de statut parce qu'elle est devenue une bataille de matériel. Les attaques au gaz donnent à la guerre un nouveau visage « qui abolira définitivement les catégories guerrières au profit des catégories sportives ». En effet, ça n'est plus la dimension militaire qui va prévaloir mais une logique de record, en particulier celui du nombre de morts. Dès lors, la distinction classique entre civils et combattants est abolie et Benjamin remarque que le droit international perd, par conséquent, son assise principale. Il reproche à ces officiers et penseurs allemands de chercher à maîtriser le présent sans avoir compris le passé. Ils regrettent les combats d'homme à homme, de troupe à troupe, qui permettaient l'expression de l'héroïsme et donnaient à la guerre une dimension cultuelle, voire artistique. Or la technique a modifié le corps de l'homme, non seulement en lui donnant des prolongements techniques et en l'inscrivant lui-même dans cette dimension, mais aussi sur le terrain militaire dans son rapport avec la mort. La guerre a perdu son aura, l'héroïsme ne peut plus singulariser celui qui n'est plus que corps, chair, comptable dans les relevés du nombre de tués. De plus, insiste Benjamin ce que Jünger et ses amis ne comprennent pas, c'est que cette bataille de matériel, dont ils veulent bien convenir, mais aussi cette guerre éternelle, qu'ils veulent retrouver et qui serait la plus haute expression de la nation 
allemande, a été perdue. Cela est spécifiquement allemand car ce sont les Allemands qui l'ont perdue et gagner ou perdre une guerre a un double sens. C'est, bien sûr, l'issue de la guerre, mais c'est aussi quelque chose, écrit-il, qui creuse un vide et affecte la réalité. " La guerre reste aux mains du vainqueur, elle échappe au vaincu »; ce qui signifie que « le vainqueur se l'approprie, en fait son bien, le vaincu en est dépossédé, il doit vivre sans elle. » Il ajoute qu'il aurait fallu que le vaincu veuille « se représenter ne serait-ce qu'un instant ce qui avait été perdu, au lieu de s'y cramponner convulsivement ». Pour l'Allemagne, toute la dimension du rapport à la question si cruciale de la nature s'est trouvée bouleversée par cette guerre d'un type nouveau. Et c'est ce qu'il aurait fallu être capable de saisir. « Disons-le en toute amertume : devant le paysage livré à la mobilisation totale, le sentiment allemand de la nature a pris un essor inattendu. Les génies de la paix qui s'y étaient si voluptueusement installés avaient été évacués, et aussi loin que portait le regard par-delà les tranchées, tout le terrain alentour offrait le visage même de l'idéalisme allemand, chaque entonnoir de grenade était un problème, chaque enchevêtrement de barbelés une antinomie, chaque pointe de fer une définition, chaque explosion une position de principe, et le ciel par là-dessus était, le jour, l'intérieur cosmique du casque d'acier, la nuit, la loi morale au-dessus de toi. La technique, avec des lignes de feu et des réseaux de tranchées, a voulu reproduire les traits héroïques de l'idéalisme allemand. Elle s'est fourvoyée. Car les traits qu'elle croyait héroïques étaient en fait hippocratiques, c'étaient les traits de la mort. Profondément pénétrée de sa propre abjection, elle modela ainsi le visage apocalyptique de la nature, la réduisit au silence, alors qu'elle était précisément la force qui aurait pu faire accéder la nature au langage. La guerre, cette guerre métaphysique et abstraite dont se réclame le nouveau nationalisme, n'est rien d'autre qu'une tentative pour faire de la technique la clef mystique permettant de résoudre immédiatement le mystère d'une nature comprise sur le mode idéaliste, au lieu d'utiliser et d'éclaircir ce mystère par le détour d'une organisation humaine. » Cette guerre, qui n'est pas plus l'éternelle guerre de ces nouveaux nationalistes que la dernière des pacifistes, mais, conclut Benjamin "l'unique, l'effrayante et dernière chance que nous ayons de corriger l'incapacité des peuples à ordonner leurs rapports mutuels, conformément à la relation qu'ils instaurent, par la technique, avec la nature. Si cette correction échoue, des millions de corps humains seront 
certes déchiquetés et dévorés par le gaz et l'acier ». Car la technique n'est pas nécessairement «un fétiche du déclin mais [peut-être] une clef du bonheur ». Ainsi la technique n'est pas univoque, ce qui aliène peut libérer. Il faut refuser à la guerre sa magie, sinon le retour de l'aura, conjugué à la technique, produira le fascisme. Dans cette perspective, le chef, le dirigeant, le Führer ne sera pas ce souverain en qui « s'unissent les pleins pouvoirs législatifs et exécutifs », cet Un, de droit divin, inscrit symboliquement, mais l'un quelconque, semblable et reproductible, pourvu d'une aura elle-même reproductible, aléatoire, que les techniques de la communication façonneront.

«Combien de temps nous faut-il encore attendre avant que les autres aussi deviennent des pacifistes ? », écrit Freud à Einstein. En somme, « tout ce qui promeut le développement culturel travaille du même coup contre la guerre ». Mais cette conclusion passe par-dessus quelques apories qu'il relève. Ainsi ce qui va dans le sens de la culture, l'intellect dominant la vie pulsionnelle et l'intériorisation du penchant à l'agression créent une aversion pour la guerre qui n'est pas qu'intellectuelle et dont il indique qu'elle n'est pas exempte de danger. Paradoxalement, ce mouvement, préjudiciable à la fonction sexuelle, peut mener à l'extinction de l'espèce humaine, ce qui serait une figure inédite de la pulsion de mort. Le retour de la violence guerrière ne sera-t-il pas à la hauteur du renoncement exigé ? Freud propose aussi d'éduquer une couche supérieure d'hommes inaccessibles à l'intimidation et luttant pour la vérité. Il réintroduit alors une distinction, cohérente selon lui, avec la division de l'humanité entre meneurs et sujets dépendants. Cette dictature de la raison, rêvée par Freud, n'est pas non plus sans danger. Là aussi, quelle sera la nature de la violence à exercer de droit pour maintenir cet ordre, qui n'est pas sans rappeler le projet platonicien ? Comment celuici se maintiendrait-il sans violence, et que faire de cette violence qui reste, qui ne se résorbe pas tout à fait dans la violence du droit? L'enjeu n'estil pas que se découvre et se distingue ce qui se perd structuralement de jouissance dans sa différence avec ce que distribue l'organisation du lien social ? La question qu'ouvre la psychanalyse est peut-être que le savoir sur cette perte peut permettre de donner sa place à cette dimension qui fut celle du sacré, un sacré cette fois-ci laïque, à quelque chose dont nous nous séparons en le sachant, en le voulant, afin de maintenir la possibilité 
de la séparation. Faute de ce sacrifice pour chacun, nous risquons alors collectivement de devoir « sacrifier aux dieux obscurs ». 